\title{
AULA ABIERTA: UN ACERCAMIENTO A LAS ESTRATEGIAS DIDÁCTICAS UTILIZADAS EN LA EXPERIENCIA CURRICULAR
}

\author{
Nayibe Tabash Blanco*
}

RESUMEN

\begin{abstract}
Aula abierta es una experiencia de flexibilización y una estrategia curricular innovadora del sistema educativo en I y II ciclos, que legitima el derecho a la educación de niños, niñas y jóvenes excluidos del sistema educativo y en riesgo de deserción. El presente artículo muestra el análisis de las guías autoformativas que se utilizan en el proyecto Aula abierta, así como las fortalezas y debilidades que tienen los docentes en relación con las estrategias didácticas que aplican en su labor diaria.
\end{abstract}

PALABRAS CLAVES: EDUCACIÓN * NIÑEZ * JUVENTUD * DESERCIÓN * ESTRATEGIAS DIDÁCTICAS $*$ ESTRATEGIA CURRICULAR

\section{ABSTRACT}

Open classroom is an experience of flexibility and an innovative curricular strategy in the educative system throughout the I and II cycles, which legitimizes the right to education of children and young people excluded, from the educative system and in risk of desertion. The present article shows to the strengths and weaknesses that teachers have in relation to the didactic strategies they apply in their daily work.

KEYWORDS: EDUCATION * CHILDHOOD * YOUTH $*$ DESERTION * DIDACTICAL STRATEGIES $*$ CURRICULAR STRATEGY

\section{INTRODUCCIÓN}

La política educativa busca expandir las oportunidades de enseñanza, así como el acceso, la permanencia y el éxito del estudiantado que ingresa al sistema educativo costarricense. Lo anterior, es un objetivo primordial para las autoridades de gobierno en materia de educación, ya que la población estudiantil enfrenta problemas sociales que 
atentan contra la permanencia en los centros educativos. Algunos de estos problemas son la drogadicción, la prostitución, la delincuencia, el abuso sexual $y$ físico y la falta de apoyo familiar, entre otros.

Por otro lado, es necesario buscar mecanismos que permitan alcanzar cada vez más, la equidad en el sistema educativo. Para alcanzar este propósito, el Ministerio de Educación Pública ha ejecutado esfuerzos en la cobertura de la educación primaria; así el Décimo Informe del Estado de la Nación, 2003, considera que

La educación primaria sigue mostrando una cobertura alta, mayor al 100\%. En el primer ciclo las tasas son de $110,3 \%$ (bruta) y $100,3 \%$ (neta). No obstante, conforme se avanza en los ciclos educativos la cobertura disminuye. Para el segundo ciclo la cobertura fue del $88,8 \%$ (tasa neta de escolaridad) en el 2002 (Gutiérrez, 2003:106-107).

Para el año 2004 el Departamento de Estadística del Ministerio de Educación Pública, registra un $8,4 \%$ de repitencia y un $4 \%$ de deserción escolar, siendo estas cifras significativas para una sociedad que tiene como principal gestor el desarrollo de la educación.

Por su parte, el Ministerio de Educación Pública a partir del año 1998 impulsa el establecimiento de programas que brindan posibilidades educativas a la población infantil y juvenil, con el propósito de que desarrollen de manera óptima las destrezas cognitivas, sociales $y$ afectivas, para lograr una formación integral $y$ la permanencia de esta población en el sistema educativo formal. Lo anterior se evidencia como una alternativa para disminuir los índices de deserción y repetición en la educación primaria.

Uno de los programas que le ofrece a esta vulnerable población la posibilidad para continuar los procesos de aprendizaje es el proyecto denominado Aula abierta. Este proyecto se enmarca dentro de la filosofía que orienta la Política Educativa hacia el Siglo XXI $y$ tiene como objetivo plantear la adopción de estrategias que permitan brindar una oferta educativa coherente con las demandas particulares de los estudiantes con sobre-edad en I y II ciclos de la Educación General Básica. El Proyecto Aula abierta, es una experiencia de flexibilización del servicio educativo destinada a la población infantil y juvenil, en riesgo social que no ha cursado la educación primaria. Como estrategia curricular es innovadora, en tanto que recrea una oferta educativa, en el marco del sistema formal, con algunos apoyos de la educación abierta, recrea un procedimiento equitativo; toma en cuenta y adecua las posibilidades reales de este tipo de población, para cursar y concluir la educación primaria. La importancia de este proyecto se menciona en el Décimo Informe del Estado de la Nación, al afirmar que

Cuando se consideran modalidades alternativas que brindan diversas instancias para que jóvenes que salen del sistema formal concluyan la Enseñanza General Básica y el ciclo diversificado, denominadas "aulas abiertas", las cifras tradicionales de tasa bruta y neta de escolaridad sufren modificaciones importantes para cuando deseen ingresar al nivel de secundaria, donde la tasa bruta pasa del $65,6 \%$ a $75,4 \%$ y la tasa neta de $58,7 \%$ a $63,5 \%$ en el año 2003 (Gutiérrez, 2003:106-107).

El proyecto Aula abierta cuenta con recursos técnicos y económicos de la Organización Internacional para las Migraciones, el Instituto Mixto de Ayuda Social (IMAS), el Patronato Nacional de la Infancia (PANI), Fondo de las Naciones Unidas para la Infancia (UNICEF) de Costa Rica y otros órganos no gubernamentales.

El Proyecto Aula abierta se rige mediante los programas de estudio de Educación Abierta en las cuatro materias básicas y formación ciudadana, la aprobación de sus estudios es mediante la nota de presentación del postulante a las pruebas nacionales de conclusión del I y II ciclos de la Educación General Básica en las cinco materias básicas.

Entre las situaciones que el Ministerio de Educación Pública tomó en consideración como fundamento para la creación de la modalidad del proyecto Aula abierta se encuentran: 
$\diamond$ Tasas de repitencia en I y II ciclos, especialmente en los primeros grados del I ciclo, lo cual genera extra-edad, fracaso y deserción escolar.

$\diamond$ En los centros educativos de I y II ciclos. Existe gran cantidad de población estudiantil, que presenta la condición de extra-edad (edad superior a la esperada para el año que actualmente cursa) y con características físicas y psicológicas e intelectuales diferentes a las del resto de compañeros lo cual exige estrategias diferentes para su atención pedagógica.

$\diamond$ La población migrante extranjera requiere incorporarse al sistema educativo para continuar con su desarrollo personal.

$\diamond$ Existen condiciones psicosociales que afectan el rendimiento de los estudiantes en el sistema educativo formal entre ellas: trabajo infantil y adolescente, la desintegración familiar, el embarazo en la adolescencia $y$ otras, que requieren de un abordaje apropiado en la escuela.

$\diamond$ Diversos instrumentos jurídicos nacionales e internacionales, entre ellos la Declaración Universal de los Derechos Humanos, la Constitución Política de la República, la Ley Fundamental de Educación y el Código de la Niñez y la Adolescencia, garantizan el derecho a la educación de todas las personas y comprometen al Ministerio de Educación Pública a diversificar las ofertas $y$ a ejecutar acciones tendientes a la inclusión de aquellos sectores que han permanecido al margen de los beneficios de la educación.

Por otra parte, algunas de las características del proyecto Aula abierta son las siguientes:

$\diamond$ Promueve la atención personalizada, para garantizar que cada estudiante avance a su propio ritmo.

$\diamond$ Fortalece el estudio independiente de los estudiantes, para ello la población estudiantil cuenta con módulos de aprendizaje autoformativos.

$\diamond$ Resalta el trabajo en pequeños grupos, el cual permite el apoyo y el aprendizaje en forma cooperativa (Ministerio de Educación Pública, 2003).

Por su parte, el Ministerio de Educación Pública considera que

La metodología empleada en la modalidad Aula abierta, permite la adecuación de los contenidos, horarios y materiales didácticos a las necesidades de aprendizaje y a las características específicas de esta población estudiantil. [Además afirma que] A los estudiantes que conforman el Proyecto Aula abierta, se les facilita el aprendizaje mediante estrategias curriculares innovadoras, a partir de un replanteamiento de la oferta educativa en el marco del sistema formal, con el fin de que puedan cursar y concluir la Educación Primaria (Ministerio de Educación Pública, 2003: 17).

Por lo tanto, la prioridad es ofrecer espacios para que el estudiantado considere la educación como una oportunidad fundamental de progreso y no como un sistema represivo que pretende mantenerlos bajo su sombra a través de conceptos de obligatoriedad.

La población meta que atiende este proyecto está constituido por adolescentes cuyas edades oscilan entre los 13 a 15 años (condición indispensable), asociada a alguna(s) de las siguientes condiciones:

\& Persona incorporada al sistema educativo, en condición de extra-edad y que presenta una inadaptación evidente en el sistema educativo formal (se excluyen adolescentes que estén cursando el sexto grado). Ellos tienen al menos, tres años más de edad biológica que corresponde al grado que cursa, a esto se le conoce como la condición de sobre-edad escolar o extra-edad.

$\diamond$ Niños, niñas y jóvenes que nunca ingresaron a la escuela.

$\diamond$ Jóvenes desertores de I y II ciclos de la Educación General Básica.

$\diamond$ Jóvenes que desempeñan actividades laborales, formales o informales remuneradas o no. 
$\diamond$ Madres adolescentes.

$\diamond$ Estudiantes migrantes que han ingresado al país sin documentación que certifique la escolaridad aprobada.

$\diamond$ Jóvenes en "situación de calle" institucionalizados (Ministerio de Educación Pública, 2003: 25).

Además, muchos de los adolescentes que atiende este proyecto son jóvenes en condición de extrema pobreza y que habitan en zonas vulnerables, es decir, comunidades en donde se puede adquirir la droga y el alcohol en cualquier esquina. En este programa también se pueden encontrar jóvenes trabajadores de la calle. Todos ellos buscan en la educación un medio para tratar de salir de la condición económica en que se encuentran.

Para hacer efectivos los lineamientos brindados por el Ministerio de Educación Pública en el proyecto Aula abierta, es importante que el o la educadora se comprometa más con la tarea de innovar y buscar nuevas posibilidades metodológicas que hagan de la escuela un sitio agradable, que no resulte agobiante ni aburrido. La planificación de los procesos de enseñanza $y$ aprendizaje deben caracterizarse por la flexibilidad implícita en la acción pedagógica. Esto conlleva que el educador conozca una serie de estrategias metodológicas que le posibiliten su accionar dentro del aula de manera exitosa, y diseñe técnicas de estudio para aquellos estudiantes que no pueden asistir regularmente a la escuela, siempre y cuando utilicen los materiales didácticos adecuados y se establezcan las acciones que permitan el seguimiento, apoyo $y$ evaluación para tales casos.

Lo expuesto anteriormente, compromete al educador en la búsqueda de métodos atractivos, que le provean al estudiantado mayor seguridad en la comprensión y construcción del conocimiento.

A su vez, la planificación de las estrategias didácticas debe adaptarse al nivel cognoscitivo, afectivo y psicomotor de cada estudiante. En este sentido, el reto que tiene el educador es mantener al alumnado motivado y comprometido con su propio aprendizaje, por lo que la investigación educativa debe ser constante $y$ sistemática.
Sobre la base de lo expuesto anteriormente, es que surgió la necesidad de realizar una investigación que tuviera como objetivo analizar las estrategias didácticas que emplean los educadores que forman parte del proyecto Aula abierta, por un lado para tener una visión más clara de la oferta curricular que prevalece en las instituciones educativas, $y$ por otro para elaborar una guía didáctica que oriente los procesos de enseñanza y aprendizaje de los educadores que participan en este proyecto, con el fin de lograr en el estudiantado procesos de formación desde una perspectiva integradora.

La investigación se planteó los siguientes objetivos específicos:

\& Caracterizar las estrategias didácticas presentes en los módulos autoformativos que utilizan los docentes que participan en el Proyecto Aula abierta.

$\diamond$ Determinar los estilos de aprendizaje de la población estudiantil que participan del Proyecto Aula abierta.

$\diamond$ Identificar las estrategias didácticas presentes en el planeamiento didáctico que elaboran los educadores.

\& Establecer la relación entre las estrategias didácticas presentes en los módulos autoformativos y los estilos de aprendizaje del estudiantado.

$\diamond$ Determinar los problemas que enfrentan los estudiantes en la mediación pedagógica.

\section{PROCEDIMIENTO METODOLÓGICO}

La investigación se orienta dentro del enfoque cualitativo, ya que toma como fuente diferentes realidades, para buscar, descubrir y analizar la cultura, así como el comportamiento de los seres humanos desde el punto de vista de quien investiga.

Por otra parte, el presente estudio incorpora elementos propios de la investigación descriptiva, ya que en él se recopila información sobre diversas categorías de análisis de diferentes sujetos, con el fin de establecer la relación entre ellos y posteriormente describir los hallazgos encontrados (Taylor y Bodgan, 1998:43). 
La investigación se realizó en dos instituciones educativas de carácter público, ubicadas en la provincia de San José en los circuitos 03 y 10. La población que constituye el estudio está compuesta por cincuenta $y$ un estudiantes $y$ dos maestras de educación primaria que trabajan en el Proyecto Aula abierta.

Con el propósito de efectuar un análisis válido y confiable de la información que se recopiló, Buendía (2000) menciona que se requiere de la identificación de categorías de análisis entendidas como "una conceptualización realizada a partir de la conjunción de elementos que tienen características comunes" (2000:84). Para esta investigación se definieron las siguientes categorías:

Planificación curricular: "Es una planificación cuidadosa y sustentada en diagnósticos previos" (Molina, 2000: 35).

Estrategias didácticas: "Todas aquellas etapas o fases seguidas en una secuencia de enseñanza, sustentadas en desarrollos teóricos $y$ validadas desde el punto de vista de los resultados obtenidos" (Maya, 1998:54).

Estilos de aprendizaje: "Es el compuesto de factores cognitivos, afectivos y fisiológicos que sirven como indicadores relativamente estables de cómo el aprendiz percibe, interactúa con su entorno y responde a él" (Pérez, 2002:12).

La información se recolectó utilizando los siguientes instrumentos:

a. Observación participante y notas de campo de la realidad educativa de aula. Este instrumento permitió la recolección de información de las estrategias didácticas que aplicaban las educadoras en el desarrollo de sus lecciones y los estilos de aprendizaje de los estudiantes. El proceso de observación se suspendió en el momento en que la investigadora consideró que se había llegado a un punto de saturación, que es cuando se empiezan a observar los mismos indicadores $y$ ejemplos en las aulas sin datos relevantes que marquen alguna diferencia o dato significativo. Se realizaron un total de veinte observaciones.

b. Cuestionario dirigido a las educadoras. Este recurso facilitó la obtención de información acerca de la metodología que utilizaban las educadoras en sus lecciones y sobre los elementos estructurales de la planificación curricular.

c. Un cuestionario dirigido a los estudiantes. Este instrumento brindó información acerca de los estilos de aprendizaje con los cuales se identificaba cada estudiante.

d. Entrevista dirigida a las educadoras. Este instrumento permitió la recolección de información relacionada con los objetivos y su relación con los procesos de enseñanza $y$ aprendizaje; las actividades de aprendizaje presentes en cada módulo autoformativo; ventajas $y$ desventajas que presentan los módulos autoformativos; los estilos de aprendizaje de la población estudiantil y los mecanismos que incorpora cada educadora en su labor cotidiana, para brindarle seguimiento a cada estudiante.

e. Entrevista dirigida a los estudiantes. La entrevista recopila información acerca de las actividades que realizan las educadoras en las lecciones; las actividades y recursos que al estudiantado le agradaría que las educadoras apliquen en sus lecciones; la utilidad de los módulos autoformativos; la evaluación del trabajo de clase; las ventajas y desventajas del Proyecto Aula abierta.

f. Hoja de cotejo para analizar los módulos autoformativos. Con este instrumento se recolectó información sobre la redacción de los objetivos específicos que se incluyen en cada uno de los módulos autoformativos; la relación entre los objetivos específicos $y$ los contenidos; la coherencia entre las actividades $y$ los objetivos específicos y por último la coherencia entre las actividades y los objetivos.

\section{RESULTADOS OBTENIDOS}

Para realizar el análisis de los datos se procedió a realizar la triangulación, para ello se tomó en cuenta la información que se obtuvo de los diferentes instrumentos que se aplicaron a los diferentes actores.

Los resultados más relevantes son los siguientes: 
$\diamond$ Las estrategias didácticas que predominan en las guías autoformativas son las de transmisión y las de elaboración de la información. En la primera estrategia el estudiante reproduce la información de manera escrita, para ello debe resolver ejercicios, cuestionarios y problemas. La segunda estrategia se caracteriza por la variedad de ejemplos $y$ ejercicios que le brindan al estudiantado para que estos adquieran los conocimientos deseados.

$\diamond$ Las educadoras y la población estudiantil coinciden en que las actividades presentes en las guías autoformativas les ayudan en los aprendizajes de las diferentes áreas temáticas, porque les permiten comprender mejor los temas debido a que en estas guías se presentan resúmenes de la materia que se desea desarrollar, así como ejercicios que conllevan a la aplicación de la misma.

४ En cuanto a las estrategias de evaluación de los aprendizajes, las guías presentan actividades que favorecen la evaluación formativa, pues les permite resolver diferentes ejercicios que luego son revisados en forma oral por las educadoras. Además, se presenta la evaluación sumativa debido a que el estudiantado debe realizar una prueba para aprobar cada una de las asignaturas que recibe.

$\diamond \mathrm{El}$ estilo de aprendizaje global, es el que se promueve en las actividades que desarrollan las educadoras en las lecciones. En este tipo de aprendizaje los estudiantes aprenden más fácilmente cuando entienden primero el concepto y luego pueden concentrarse en los detalles, pueden trabajar con distractores, la población estudiantil constantemente se moviliza de un lugar a otro e incluso la organización de los pupitres no tiene una organización definida. Sin embargo, la investigación destaca que los estudiantes poseen un estilo de aprendizaje analítico y divergente. En el estilo analítico la información es brindada paso a paso en forma acumulativa $y$ en secuencia, de manera tal que se comprendan los conceptos.

$\diamond$ En el estilo de aprendizaje divergente primero se captan las experiencias en forma real y concreta para luego reflexionar sobre ellas $y$ comprender el significado de la materia en estudios, se aprende escuchando, compartiendo ideas $y$ analizando situaciones desde diferentes perspectivas. Por lo tanto es posible mencionar que no existe relación entre el estilo de aprendizaje que las educadoras promueven con las diferentes actividades que aplican en las lecciones y con la población estudiantil.

$\diamond$ Existe relación entre las estrategias didácticas presentes en las guías autoformativas $y$ las estrategias aplicadas por las educadoras en las lecciones, ya que en ambos casos se utilizaron las de transmisión. Entre las actividades que promovieron son el uso del libro de texto, la comprensión de lectura, resolución de prácticas y fichas, explicación oral de las temáticas abordadas y trabajo en grupo, entre otras.

$\diamond$ Las estrategias didácticas de integración grupal y las orientadas a buscar nueva información, únicamente se mencionan como actividades extracurriculares.

$\diamond$ Según acuerdo del Consejo Superior de Educación, la estructura del planeamiento didáctico en el proyecto Aula abierta, se rige por lo establecido en los lineamientos del sistema formal, donde los contenidos que se incorporan son los que se presentan en las guías autoformativas de cada nivel y además se deben tomar en cuenta los temas transversales. Sin embargo, se evidenció que las educadoras no realizan un planeamiento didáctico, en su lugar elaboran crónicas semanales las que se refieren al avance de los estudiantes con respecto a las guías autoformativas.

$\diamond$ Las guías autoformativas presentan un enfoque educativo metodológico con estrategias didácticas que favorecen el estilo de aprendizaje global, en este se presenta primero el concepto y luego los detalles, no favorecen la comprensión del contenido en forma más profunda, mientras que los estudiantes señalan una inclinación hacia los estilos de aprendizaje analítico y divergente.

$\diamond$ Entre los principales problemas que enfrenta la población estudiantil en la mediación pedagógica se encuentran: discriminación por parte de la comunidad estudiantil que no forma parte del proyecto Aula abierta, 
indisciplina, monotonía en el trabajo con las guías autoformativas $y$ la falta de interés por parte de la población estudiantil al realizar el trabajo diario, la falta de planificación de la labor que deben de realizar las educadoras con la población estudiantil. Por otra parte, es necesario que las educadoras lleven un control del avance de cada estudiante.

४ La metodología empleada en el trabajo de aula es tradicional, falta implementar actividades de motivación e investigación, que les proporcione mayor información a los estudiantes, en relación con los contenidos que se van a desarrollar. Además, es necesario que se realicen actividades que complementen las que se ofrecen en las guías autoformativas tales como utilizar juegos para la comprensión de los contenidos, realizar trabajos grupales, elaborar mapas conceptuales, hacer visitas de campo e integra las artes plásticas y la música, en las diversas actividades que realizan las educadoras en el aula escolar.

$\diamond$ El perfil que describe el Ministerio de Educación Pública para el educador o la educadora que atiende el proyecto Aula abierta establece que el docente o la docente debe ser sensible ante la problemática del estudiante $y$ además deben ser personas proactivas, que promuevan su propio desarrollo personal y profesional. Sin embargo, las educadoras participantes en la investigación carecían de elementos de investigación que le permitan indagar de manera constante y sistemática sobre las diversas situaciones que deben de enfrentar en el aula.

$\diamond$ El estudiantado expresó que les gustaría que las educadoras lleven a cabo actividades tales como: visitas de campo, excursiones, trabajos grupales, elaboración de resúmenes, experimentos, exposiciones, actos cívicos, bailes, cantos y maquetas.

$\diamond$ La población estudiantil afirmó que los aprendizajes que reciben en el aula son importantes para su vida, principalmente porque les permite ser mejores personas así como comprender más y obtener mayor conocimiento.

\& La población estudiantil manifestó que para facilitar y comprender mejor los contenidos se debe incluir en las guías autoformativas elementos tales como: prácticas intensivas, mayores explicaciones de los temas por tratar, el uso del color en las guías ya que estas se presentan en blanco y negro, resolución de rompecabezas, juegos de palabras. Además afirmaron que era necesario que se realizaran las actividades extracurriculares que vienen descritas en las guías como visitas de campo, elaborar investigaciones en forma individual y grupal, utilizar los mapas y la calculadora.

$\diamond$ Los y las estudiantes mencionaron que entre las dificultades que afrontaron en el aula regular y que les hizo incorporarse al proyecto Aula abierta se encuentran:

Falta de comprensión de los contenidos, exámenes muy difíciles, mucho trabajo en la pizarra y gran cantidad de materia por copiar, maltrato por parte de los compañeros, problemas con el educador o la educadora, trabajar para ayudar económicamente a la familia, tener que usar uniforme, llamadas de atención constante por parte de la educadora o el educador, gran cantidad de estudiantes por grupo, lo que impedía que la maestra o el maestro brindara una atención individual.

$\diamond$ La población estudiantil mencionó que el proyecto Aula abierta tiene las siguientes ventajas: horario flexible, hay comprensión por parte de las educadoras en cuanto a la situación socioeconómica y su nivel académico, el título lo obtienen en forma más rápida y el aprendizaje de los diferentes contenidos les resulta más fácil, no tienen que llevar uniforme, hay mayor facilidad para resolver los exámenes ya que hay una atención más individualizada y hay mayor afinidad con los compañeros debido a que los grupos son más pequeños.

$\diamond$ Entre las desventajas que tiene el Proyecto Aula abierta el estudiantado manifestó: Existe discriminación hacia ellos por parte de la comunidad estudiantil, lo cual permite que los culpen de todo lo malo que sucede en la institución educativa, existe mucha 
indisciplina de los estudiantes, los procesos de enseñanza $y$ aprendizaje se tornan muy aburridos, debido a que el tiempo que se aprovecha en el aula es muy poco y siempre se trabaja con los mismos libros.

$\diamond$ En relación con las guías autoformativas es importante mencionar que estas son una colección de diez libros elaborados por el Instituto Costarricense de Enseñanza Radiofónica. Surgen como una respuesta a las necesidades de la población estudiantil que no han concluido la Enseñanza General Básica.

Al realizar el análisis de estas guías se evidenció lo siguiente:

$\diamond$ Ninguna de las guías autoformativas contienen objetivos generales, todas incluyen objetivos específicos redactados en primera $y$ tercera persona, no existe uniformidad en cuanto a la redacción de los objetivos en una y otra guía.

$\diamond$ Todas las guías presentan los contenidos respectivos, pero al igual que en los objetivos no existe uniformidad en su presentación, ya que en algunas el formato contiene el nombre y el número de la unidad, objetivos, contenidos y actividades. En otras, los contenidos se titulan o engloban en un nombre que se le ha asignado a cada unidad, así mismo los temas se desprenden de las actividades por desarrollar. Es importante mencionar que los contenidos no se enumeran en un listado, sino que se especifican en unos párrafos que están antes de la explicación del desarrollo de las actividades.

$\diamond$ Todas las guías contienen actividades extracurriculares, estas tienen coherencia con los objetivos específicos y contenidos que se presentan.

$\diamond$ Las actividades que se presentan en las guías autoformativas evidencian muy poca integración con otras disciplinas. La guía del área de Español es en la que más se presenta la integración con otros contenidos de materias tales como Ciencias, Estudios Sociales y del área artística por ejemplo: canciones, chistes, trabalenguas y dramatizaciones que le permiten al estudiante desarrollar habilidades linguísticas y de expresión corporal.

$\diamond$ En la mayoría de las guías autoformativas se percibe una integración de los temas transversales en las actividades que los estudiantes deben realizar.

$\diamond$ En todas las guías se muestran actividades que evalúan la comprensión de lectura en los niveles literal, inferencial, apreciativo y reorganizacional, el nivel recreativo no se contempla en ninguna de las actividades.

$\diamond$ Se presentan actividades de transmisión, porque la información está en un orden adecuado y provoca que el estudiante reproduzca la información de manera escrita. El estudiante lee la información y para demostrar que la comprende debe realizar tareas como contestar preguntas, rellenar espacios, resolver problemas, completar asociaciones, resolver crucigramas, identificar dibujos y realizar ejercicios de caligrafías, entre otros, todos en forma escrita.

$\diamond$ Ninguna de las guías tienen actividades de motivación, lo único que se presentan son mapas y esquemas en cada uno de los contenidos.

$\diamond$ Ninguna de las guías describe técnicas didácticas que estimulen la expresión creativa $y$ crítica.

$\diamond$ Las guías autoformativas incluyen actividades que estimulan al estudiante a resolver problemas de la vida cotidiana.

Una segunda etapa de la investigación consistió en la elaboración de un módulo didáctico dirigido al personal docente que trabaja en el Proyecto Aula abierta.

El módulo tiene el objetivo dar a conocer lineamientos metodológicos para fortalecer los procesos de enseñanza y aprendizaje de la población estudiantil. Se espera que este aporte constituya un recurso didáctico para el educador o educadora y que este encuentre en el módulo una ventana hacia otras innovaciones educativas, las cuales respondan a lograr mejoras en la calidad de la educación costarricense.

Los hallazgos encontrados que alimentaron la propuesta son los siguientes: 
a. La metodología empleada en el trabajo de aula es tradicional.

b. Las educadoras no elaboran un planeamiento didáctico para ejecutar sus lecciones.

c. Las estrategias didácticas que presentan las guías autoformativas y las que aplican las educadoras en sus lecciones no toman en cuenta los diferentes estilos de aprendizaje de los estudiantes.

d. Falta ejecutar actividades de motivación e investigación, que le proporcionen mayor información a los estudiantes en relación con los contenidos que van a desarrollar.

El módulo didáctico se divide en cuatro capítulos. El primer capítulo se denomina "El proyecto Aula abierta del Ministerio de Educación Pública: definición, población meta, objetivo primordial, enfoque pedagógico, perfil del educador, y estrategias generales". En este capítulo se ofrece una descripción detallada del proyecto, con el fin de que los educadores y las educadoras fundamenten y respalden su práctica docente.

El segundo capítulo se denomina "El planeamiento didáctico: concepto, elementos, importancia y recomendaciones". En este se describen aspectos teóricos y prácticos acerca del concepto de planeamiento educativo, así como los elementos que lo componen, su importancia y recomendaciones que los educadores o educadoras pueden incluir en su labor diaria.

El tercer capítulo se refiere a "Los estilos de aprendizaje: Teoría de las inteligencias múltiples y la inteligencia emocional". Se analizan aspectos teóricos y prácticos de los estilos de aprendizaje, algunas clasificaciones e instrumentos que identifican mejor cada estilo. Así como, la perspectiva de especialistas que han elaborado teorías, en relación con las formas de aprender $y$ la inteligencia propiamente dicha.

En el cuarto capítulo se citan y desarrollan estrategias metodológicas, técnicas de motivación, técnicas grupales y técnicas de evaluación que los educadores y las educadoras que laboran en el Proyecto Aula abierta pueden emplear en su labor docente como complemento a las guías didácticas. Se presentan aspectos teóricos y prácticos relacionados con las estrategias didácticas, tipologías y ejemplos de estrategias que los educadores o educadoras pueden realizar en el salón de clase.

\section{CONSIDERACIONES FINALES}

Partiendo de que el Proyecto Aula abierta es una experiencia de flexibilización curricular, destinado a poblaciones de estudiantes que se encuentran en riesgo social y cuyo propósito es permitirles que culminen su formación básica, para que estén en capacidad de continuar con sus estudios en cualquiera de las diversas ofertas del sistema educativo costarricense, es importante que los educadores y las educadoras que participen en este proyecto sean concientes de que el mismo representa un verdadero reto, ya que además de las labores propias de su especialidad profesional, el docente o la docente debe mantener al estudiantado motivado y comprometido con su propio aprendizaje. Este propósito se cumplirá en la medida en que el personal docente planifique sus actividades y que estas a su vez, se adapten al nivel cognoscitivo de la población estudiantil y a las necesidades del contexto en que se encuentra inmersa la población estudiantil. De aquí la importancia de aplicar diversas actividades que sean interesantes, pertinentes y relacionadas con las temáticas de estudio, especialmente planificadas para la asimilación de formas o saberes culturales, que resultan a la vez esenciales en el desarrollo y socialización de la población estudiantil y serían difíciles de asimilar de no ser por la orientación específica del educador o educadora, la cual debe estar en función de los estilos de aprendizaje que posee el estudiantado.

En síntesis, es necesario prever un planeamiento metodológico acorde con los requerimientos específicos para este tipo de población, acompañado de diagnósticos previos, con el fin de que los procesos de enseñanza y aprendizaje se tornen más significativos, $y$ a la vez se proporcione la información necesaria, se utilice una metodología más activa, se haga un adecuado tratamiento de los errores y se propicie un aprendizaje cooperativo. 


\section{REFERENCIAS BIBLIOGRÁFICAS}

Barrantes, R. Investigación un camino al conocimiento. Un enfoque cualitativo y cuantitativo. San José, Costa Rica, Cr: EunED. 2000 .

Buendía, L. y otros. Métodos de investigación en psicopedagogía. Madrid, España: Mc Graw Hill. 2000.

Bonilla, E. y otros. Más allá del dilema de los métodos. Santa Fe de Bogotá, DC.: Uniandes. 1997.

Campos, S. Introducción a la Psicología Social. San José, Costa Rica. EUNED. 2003.

Carranza, T. y otros. "El proceso didáctico en la enseñanza de la Historia”. Tesis de grado. Universidad de Costa Rica. 2002.

Cerda, H. Los elementos de la investigación, cómo reconocerlos, diseñándolos y construirlos. Santa Fe de Bogotá, DC: El Buho LTDA. 1991.

Coll, C. y otros. Los contenidos de la reforma enseñanza y aprendizaje de conceptos, procedimientos y actitudes. España. Editorial Santillana. 1992.

Ferreira, O. y otros. Técnicas grupales. Elementos para el aula flexible. Buenos Aires, Argentina. Ediciones Novedades Educativas. 1997.

Gallo, C. Evaluación de los procedimientos. Guatemala. Editorial Piedra Santa. 1998.

Goleman, D. La inteligencia emocional. México. Vergara Editor SA. 1997.

Good, T. y otros. Psicología educativa contemporánea. México. Mc Graw Hill. 1996. /Internacional Editores, SA de CV.
Gutiérrez, M. Décimo Informe del Estado de la Nación. San José, Costa Rica. 2003.

Hernández, R. Manual operativo para estimulación y crecimiento del desarrollo del niño. San José, Costa Rica. EUNED. 2002.

Hurlock, E. Desarrollo del niño. México. Mc Graw Hill Editores. 1991.

Matlos, A. Compendio de didáctica general. Buenos Aires, Argentina. Editorial Kapeluz. 1979.

Maya, A. La escuela líder. Una institución inteligente para mejorar la calidad de la educación. San José, Costa Rica. SIMED-MEP. 1998.

Ministerio de Educación Pública. Proyecto Aula abierta. San José, Costa Rica. Editorial Costa Rica. 2003.

Mello, I. El proceso didáctico. Buenos Aires, Argentina: Editorial Kapeluz SA. 1974.

Molina, T. Planeamiento didáctico. Buenos Aires, Argentina. Editorial Kapeluz. S.A. 1997.

Molina, Z. Planeamiento didáctico: fundamentos, principios y procedimientos para el desarrollo. San José, Costa Rica. EUNED. 2000.

Montoya, I. "Lineamientos metodológicos para la aplicación de adecuaciones curriculares en II ciclo de la Enseñanza General Básica”. Tesis de grado. Universidad de Costa Rica. 2003.

Navarro, A. Evaluación de los aprendizajes en la escuela primaria: una nueva visión. Cartago, Costa Rica. Imprenta Bogantes. 2002.

Pérez, C.R. El constructivismo en los espacios educativos. Cartago, Costa Rica. Impresora Obando SA. 2002. 
Picado, G. Didáctica general. San José, Costa Rica. EUNED. 2001.

Regina y otros. "El proyecto Aula abierta y su incidencia en el proceso educativo". Tesis de grado. Universidad de Costa Rica. 2005.

Solano, A.J. Educación y aprendizaje. Cartago, Costa Rica. Impresora Obando SA. 2002.
Taylor, S. y otros. Introducción a los métodos cuantitativos de investigación. Barcelona, España. Paidós Ibérica SA. 1998.

Woolfolk, A. Psicología Educativa. México. Pentice-Hall Hispanoamericana. 1990. 
